\title{
Spontaneous subarachnoid hemorrhage of unknown origin: hospital course and long-term clinical and angiographic follow-up
}

\author{
Ali M. Elhadi, MD, PhD,,2 Joseph M. Zabramski, MD,,3 Kaith K. Almefty, MD, ${ }^{1}$ \\ George A. C. Mendes, MD, ${ }^{2}$ Peter Nakaji, MD, ${ }^{1}$ Cameron G. McDougall, MD, ${ }^{1}$ \\ Felipe C. Albuquerque, MD, ${ }^{1}$ Mark C. Preul, MD, ${ }^{2}$ and Robert F. Spetzler, MD'
}

'Division of Neurological Surgery; ${ }^{2}$ Neurosurgical Research Laboratory, Barrow Neurological Institute, St. Joseph's Hospital and Medical Center, Phoenix; and ${ }^{3}$ Division of Neurological Surgery, Scottsdale Healthcare Osborn, Scottsdale, Arizona

\begin{abstract}
OBJECT Hemorrhagic origin is unidentifiable in 10\%-20\% of patients presenting with spontaneous subarachnoid hemorrhage (SAH). While the patients in such cases do well clinically, there is a lack of long-term angiographic followup. The authors of the present study evaluated the long-term clinical and angiographic follow-up of a patient cohort with $\mathrm{SAH}$ of unknown origin that had been enrolled in the Barrow Ruptured Aneurysm Trial (BRAT).

METHODS The BRAT database was searched for patients with SAH of unknown origin despite having undergone two or more angiographic studies as well as MRI of the brain and cervical spine. Follow-up was available at 6 months and 1 and 3 years after treatment. Analysis included demographic details, clinical outcome (Glasgow Outcome Scale, modified Rankin Scale [mRS]), and repeat vascular imaging.

RESULTS Subarachnoid hemorrhage of unknown etiology was identified in 57 (11.9\%) of the 472 patients enrolled in the BRAT study between March 2003 and January 2007. The mean age for this group was 51 years, and 40 members $(70 \%)$ of the group were female. Sixteen of 56 patients $(28.6 \%)$ required placement of an external ventricular drain for hydrocephalus, and 4 of these subsequently required a ventriculoperitoneal shunt. Delayed cerebral ischemia occurred in 4 patients $(7 \%)$, leading to stroke in one of them. There were no rebleeding events. Eleven patients were lost to followup, and one patient died of unrelated causes. At the 3 -year follow-up, 4 (9.1\%) of 44 patients had a poor outcome (mRS > 2), and neurovascular imaging, which was available in 33 patients, was negative.
\end{abstract}

CONCLUSIONS Hydrocephalus and delayed cerebral ischemia, while infrequent, do occur in SAH of unknown origin. Long-term neurological outcomes are generally good. A thorough evaluation to rule out an etiology of hemorrhage is necessary; however, imaging beyond 6 weeks from ictus has little utility, and rebleeding is unexpected.

http://thejns.org/doi/abs/10.3171/2014.10.JNS14175

KEY WORDS angiographically negative; subarachnoid hemorrhage; BRAT; angiographic follow-up; hospital stay; vascular disorders; SAH of unknown origin

$\mathrm{N}$ ONTRAUMATIC subarachnoid hemorrhage (SAH) accounts for approximately $5 \%$ of all stroke admissions in the United States each year, with an estimated incidence of $10-15$ cases per 100,000 population. ${ }^{1,16}$ Aneurysms and other vascular abnormalities are well-documented causes of nontraumatic SAH; however, in 10\%-
$20 \%$ of cases, no underlying vascular etiology is found, and the source of hemorrhage remains unknown. ${ }^{5,15,18,26,28}$ Patients with an SAH of unknown etiology typically have a benign hospital course and are discharged to home in good condition.

While the clinical outcome of these patients has been

ABBREVIATIONS AVM = arteriovenous malformation; BRAT = Barrow Ruptured Aneurysm Trial; $C T A=C T$ angiography; $C T N=C T$ negative; $D S A=$ digital subtraction angiography; EVD = external ventricular drain; GOS = Glasgow Outcome Scale; HH = Hunt and Hess; IQR = interquartile range; IVH = intraventricular hemorrhage; LOS = length of hospital stay; MRA = MR angiography; $\mathrm{mRS}=$ modified Rankin Scale; $\mathrm{PMH}=$ perimesencephalic hemorrhage; $\mathrm{SAH}=$ subarachnoid hemorrhage; VP = ventriculoperitoneal.

SUBMITTED January 21, 2014. ACCEPTED October 22, 2014.

INCLUDE WHEN CITING Published online December 19, 2014; DOI: 10.3171/2014.10.JNS14175.

DISCLOSURE The authors report no conflict of interest concerning the materials or methods used in this study or the findings specified in this paper. 
well documented, the results of long-term angiographic follow-up are unknown. We undertook this study to examine long-term clinical and angiographic follow-up in patients with SAH of unknown etiology who had been identified as part of the Barrow Ruptured Aneurysm Trial (BRAT). ${ }^{17,24}$

\section{Methods}

Between March 2003 and January 2007, a total of 500 patients consented to participate and were enrolled in the Barrow Ruptured Aneurysm Trial (BRAT). Consent was erroneously obtained in 28 patients, leaving 472 patients eligible for analysis. Reasons for consent errors included events such as hemorrhage more than 14 days before presentation, age exclusions, and the ultimate determination that SAH had been caused by trauma or that SAH had not occurred at all. A prospectively collected database of the 472 patients was searched for those with no identifiable source of the hemorrhage during their initial hospitalization. The BRAT is a prospective, randomized, controlled study designed to compare the results of surgical clipping with those of endovascular coiling in the treatment of ruptured intracranial aneurysms. A description of the study, as well as outcome data at 1 and 3 years, has been published. ${ }^{17,24}$ Briefly, all patients between the ages of 18 and 80 years who had been admitted to the intensive care unit with acute nontraumatic SAH (diagnosed by CT or lumbar puncture) were eligible to participate and were included if they or their health care decision surrogate consented. Patients with traumatic SAH and those presenting to the hospital more than 14 days after hemorrhage were excluded. To maximize the comprehensive nature of the BRAT, all patients with diagnostically proven $\mathrm{SAH}$ were enrolled and continued to be tracked even if no source of hemorrhage was ever identified.

After enrollment, all patients received the same protocol of care. Initial evaluation included CT angiography (CTA) or conventional digital subtraction angiography (DSA). The latter was performed in all patients whose CTA was negative for the source of the SAH. If no source for the hemorrhage was found on admission imaging, angiography was repeated 1 week later. Patients in these cases also underwent MRI and MR angiography (MRA) of the brain and cervical spine. If no responsible lesion was detected during the initial hospitalization, patients underwent outpatient follow-up vascular imaging (CTA, MRA, or catheter-based angiography) at 4-6 weeks posthemorrhage. For the purposes of the present study, only those patients in whom both the inpatient and the early outpatient vascular imaging studies failed to reveal an aneurysm or other vascular abnormality were considered to have no identifiable source of SAH.

A complete admission history, a physical, and standard screening laboratory work were performed in all patients. The Glasgow Coma Scale score, Hunt and Hess (HH) grade, and Fisher grade were calculated on admission. Independent neuroradiologists analyzed all imaging data.

A dedicated research nurse practitioner acted as the study coordinator, monitored patient accrual and randomization, and was responsible for collecting follow-up data and assessing modified Rankin Scale (mRS) and Glasgow Outcome Scale (GOS) scores. Patients were asked to return for follow-up at 6 months, 1 year, and 3 years after treatment. At the 3-year follow-up visit, patients were asked to undergo repeat angiographic evaluation; the type of imaging performed (DSA, CTA, or MRA) was left to the discretion of the treating physician.

\section{Data Analysis}

All admission head CT scans were reviewed, and patients were assigned to 1 of 3 SAH groups: 1) the CT was negative (CTN), but SAH was confirmed by lumbar puncture; 2) classic hemorrhage pattern consistent with aneurysmal rupture; and 3) perimesencephalic hemorrhage $(\mathrm{PMH})$, which was defined according to published criteria $^{20,21}$ and included a focus of SAH ventral to the brainstem with limited or no evidence of hemorrhage in the basal, interhemispheric, or sylvian cisterns. Patients with no hemorrhage detected on CT scans but who had xanthochromia on lumbar taps were classified as having CTN SAH. The extent of SAH on admission CT was graded using a modified Fisher scale (Table 1), with intraventricular hemorrhage (IVH) documented separately as present or absent. ${ }^{6,15}$ Delayed cerebral ischemia was defined as the occurrence of local neurological impairment or a decrease of at least 2 points on the Glasgow Coma Scale not attributed to other causes by means of clinical assessment, CT or MRI of the brain, and appropriate laboratory studies. ${ }^{27}$ Functional status was based on GOS and mRS scores. ${ }^{2,13}$

\section{Statistical Analysis}

Demographic data and bleeding patterns were analyzed using descriptive statistical analysis, outcomes for bleeding pattern groups were compared using a t-test, and length of hospital stay (LOS) was analyzed using the Mann-Whitney U-test. Regression statistics and ANOVA were used for evaluating multivariate analysis. Statistical significance was determined by $\mathrm{p}<0.05$.

\section{Results}

Among the 472 patients eligible for analysis, 57 patients had no source of hemorrhage identified during their initial hospitalization. One of these patients, a 67-year-old woman with a Fisher Grade 2 hemorrhage, had a small (2-3 $\mathrm{mm}$ ) basilar trunk aneurysm identified on CTA during an outpatient follow-up 6 weeks after hemorrhage. The aneurysm was clipped without incident.

In the remaining $56(11.9 \%)$ patients, no source of hemorrhage was identified during their initial inpatient or outpatient evaluations. The mean age of this group was 51.3 years (range 19-78 years). There were 39 women and 17

TABLE 1. Modified Fisher scale

\begin{tabular}{cl}
\hline Grade & \multicolumn{1}{c}{ CT Findings } \\
\hline 1 & No evidence of SAH \\
\hline 2 & Focal or diffuse, thin SAH \\
\hline 3 & Focal or diffuse, thick SAH \\
\hline IVH & Present or absent \\
\hline
\end{tabular}


men for a female/male ratio of 2.3:1. None of the patients had previously had an SAH, intracranial aneurysm, or arteriovenous malformation (AVM). Five patients had a history of atherosclerotic disease, 6 had diabetes mellitus, and 2 had hematological disorders (thrombocytopenia).

The hemorrhage pattern on admission CT was classic in 32 patients (57\%), perimesencephalic in $13(23 \%)$, and negative (positive on lumbar puncture) in 11 patients (20\%; Table 2). The majority of patients (47 [83.9\%]) presented with an $\mathrm{HH}$ grade of I or II, whereas only $9(16.1 \%)$ had an $\mathrm{HH}$ grade of III. None of the patients had presented with an $\mathrm{HH}$ grade of IV or V.

The LOS for the 56 patients ranged from 2 to 27 days with a mean \pm standard deviation of $9.5 \pm 5.2$ days (median 8 days, interquartile range [IQR] 5.5 days). Sixteen patients showed evidence of IVH, and 13 of them (81.3\%) stayed in the hospital for 10 or more days. Of the remaining 40 patients without evidence of IVH, only 7 (17.5\%) stayed in the hospital for 10 or more days. The average LOS for patients with IVH was $14.68 \pm 5.6$ days (median 14.5 days, IQR 7 days), compared with $7.5 \pm 3.37$ days (median 7 days, IQR 2 days) for those without IVH; however, this difference did not reach statistical significance. Patients who presented with an $\mathrm{HH}$ grade of I or II had an average LOS of $8.65 \pm 5.1$ days (median 7 days, IQR 3 days), compared with $14 \pm 2.9$ days (median 15 days, IQR 5.5 days) for those who presented with an $\mathrm{HH}$ grade of III $(\mathrm{p}=0.0007)$. For the patients who had a Fisher grade of 1 or 2 on presentation, the average LOS was $7.36 \pm 3.5$ days (median 7 days, IQR 2 days), while those who had a Fisher grade of 3 stayed $13.5 \pm 5.6$ days (median 13 days, IQR 7.5 days; $\mathrm{p}<0.0001$ ).

Delayed cerebral ischemia occurred in 4 patients (7\%). All four of these patients had a classic hemorrhage pattern on admission CT. Initial treatment consisted of hypervolemia and hypertension therapy; 2 patients (3.6\%) required endovascular treatment (angioplasty and/or intraarterial infusion). Deficits resolved in all 4 patients; however, 1 patient had a diffusion-positive stroke documented on MRI in the posterior cerebral artery distribution (Fig. 1).

Sixteen patients (28.6\%) required placement of an ex-

TABLE 2. Hemorrhage pattern and clinical grade in 56 patients

\begin{tabular}{lcccc}
\hline & \multicolumn{4}{c}{ No. (\%) } \\
\cline { 2 - 5 } Parameter & CTN SAH & PMH & Classic SAH & IVH \\
\hline Mean age in yrs & 49 & 54 & 51 & 55 \\
\hline Fisher grade & & & & \\
\hline 1 & $11(100)$ & - & - & - \\
\hline 2 & - & $13(100)$ & $12(38)$ & $3 / 25(12)$ \\
\hline 3 & - & - & $20(62)$ & $13 / 20(65)$ \\
\hline HH grade & & & & \\
\hline I & $5(45)$ & $2(15)$ & $10(31)$ & $2 / 17(12)$ \\
\hline II & $6(55)$ & $11(85)$ & $13(41)$ & $8 / 30(27)$ \\
\hline III & - & - & $9(28)$ & $6 / 9(67)$ \\
\hline IV-V & - & - & - & - \\
\hline Total no. & $11(20)$ & $13(23)$ & $32(57)$ & $16(29)$ \\
\hline
\end{tabular}

- = not applicable.

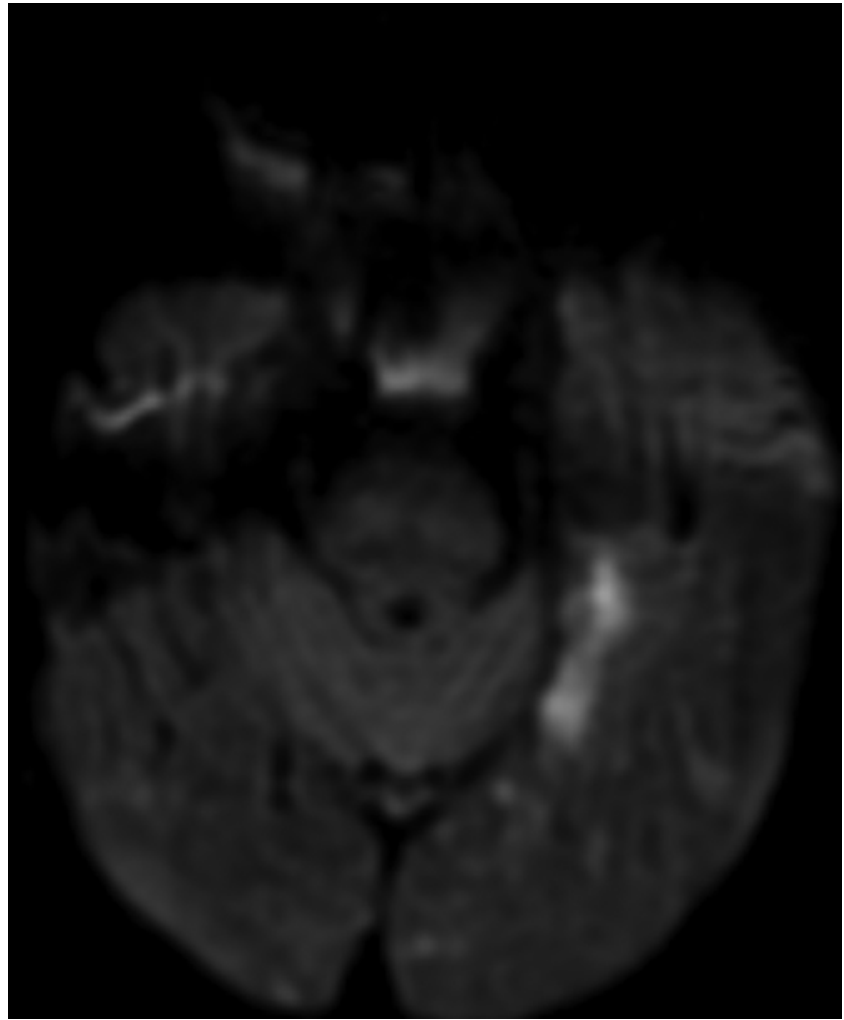

FIG. 1. Diffusion-weighted MR image demonstrating a left posterior cerebral artery ischemic stroke due to cerebral vasospasm in a patient with no identifiable source of SAH.

ternal ventricular drain (EVD) for clinical or CT evidence of hydrocephalus, and 4 of these patients subsequently required placement of a ventriculoperitoneal (VP) shunt. Three of the 4 patients who required a shunt had a classic hemorrhage pattern, and 1 had a PMH. Placement of an EVD was significantly more likely in patients with a classic hemorrhage pattern $(\mathrm{p}=0.0028$; Table 3$)$ and in those with an IVH (p < 0.0001; Table 4). A shunt was removed after 48 months in 1 patient because hydrocephalus and CSF overdrainage resolved; this patient had a classic SAH pattern on initial presentation.

Overall outcome was favorable among those who presented with no identifiable SAH source. No deaths occurred during the initial hospitalization. Fifty-two patients (93\%) were discharged to home, 4 (7\%) to inpatient rehabilitation, and none to a skilled nursing facility. Long-term outcome scores (GOS and mRS) were available in 45 of 56 patients (Table 5), as 10 patients were lost to follow-up and 1 patient died of unrelated causes at 4 months after treat-

TABLE 3. Bleeding patterns and EVD or VP shunt placement among 56 patients without an identifiable source of SAH

\begin{tabular}{ccc}
\hline Hemorrhage Pattern (no.) & No. w/ EVD & No. w/ VP Shunt \\
\hline Classic (32) & $14^{*}$ & 3 \\
\hline PMH (13) & 2 & 1 \\
\hline CTN (11) & 0 & 0 \\
\hline
\end{tabular}

* Placement of an EVD was significantly more likely in patients with a classic hemorrhage pattern $(p=0.0028)$. 
TABLE 4. Intraventricular hemorrhage and EVD or VP shunt placement among 56 patients with no identifiable SAH source

\begin{tabular}{lcc}
\hline IVH (no.) & No. w/ EVD & No. w/ VP Shunt \\
\hline With (16) & $13^{*}$ & $3 \dagger$ \\
\hline Without (40) & 3 & 1 \\
\hline${ }^{*}$ Placement of an EVD was significantly more likely in patients with an IVH ( $p$ \\
$<0.0001)$. \\
+ Placement of a VP shunt was significantly more likely in patients with an IVH \\
$(p=0.0329)$.
\end{tabular}

ment. At discharge, 29 of the 45 patients (64\%) had a good recovery (GOS Score 5), 11 (24\%) had moderate disability (GOS Score 4), and 5 (11\%) had severe disability (GOS Score 3). At the 6-month follow-up visit, the percentage of patients with good outcome (GOS Score 5) had increased to $77 \%$ (34/44 patients) and remained unchanged at the 1and 3-year follow-ups (Fig. 2). Twenty-four patients (53\%) had an mRS score of 0 or 1 on discharge (no symptoms or no significant disability despite symptoms), which increased to $75 \%(33 / 44), 84 \%$ (37/44), and 89\% (39/44) at 6 months, 1 year, and 3 years, respectively (Fig. 3). Angiographic follow-up was available in 33 of 44 patients at 3 years. The remaining 11 patients returned for clinical follow-up but declined requests for repeat imaging. Followup imaging studies included CTA in 15 patients, MRA in 17 , and DSA in 1 patient. All imaging studies were negative for aneurysm, AVM, or other vascular abnormality and were unchanged from the discharge angiograms.

The 10 patients lost to follow-up included 8 females and 2 males whose mean age was $43 \pm 13$ years. On admission, 5 of these patients had an $\mathrm{HH}$ grade of I, and 5 had an $\mathrm{HH}$ grade of II. Six patients $(60 \%)$ had a classic bleeding pattern, 2 (20\%) had PMH, and 2 (20\%) had CTN SAH. The mean LOS for this subgroup was a $9 \pm 1.4$ days (median 8 days, IQR 6 days). Three patients required placement of an EVD, but none required a VP shunt. All 10 patients were discharged to home, when 8 patients had GOS Score 5 and 2 had GOS Score 4.

\section{Discussion}

The evaluation of clinical outcome in patients with an SAH without an identifiable source has been reported; however, data on the long-term angiographic follow-up in this group of patients have been lacking. The ongoing BRAT provided an opportunity to prospectively study this group of patients. The BRAT enrolled and prospectively followed all patients with diagnostically proven SAH, even if no source of hemorrhage was ever identified. ${ }^{17,24}$ In the initial cohort of 472 patients enrolled in the BRAT, no source of hemorrhage was identified on the initial angiographic study in 100 patients (21\%). We have described our protocol for the evaluation of these patients. ${ }^{15}$ After further workup, there were 56 patients whose source of hemorrhage was not identified. The present study focuses on the long-term outcome in this group of patients with an unidentified source of SAH (defined as negative diagnostic evaluation from 4 to 6 weeks posthemorrhage).

In general, patients with an SAH of unknown etiology have a benign clinical course; however, delayed cerebral ischemia, hydrocephalus, and rebleeding have been reported. . $^{3,9,12,14,23}$ In a series of 71 patients with angiographically negative $\mathrm{SAH}$, Canhão et $\mathrm{al}^{3}{ }^{3}$ reported that $3 \%$ of patients rebled, $4 \%$ developed delayed cerebral ischemia, and 3\% had hydrocephalus that required placement of a shunt. Duong and coworkers ${ }^{4}$ described outcome at hospital discharge in a series of 87 patients with angiographically negative SAH. They reported rebleeding in $4 \%$, delayed ischemia in $4 \%$, and hydrocephalus in $14 \%$, with $3 \%$ requiring placement of a shunt. There were 2 deaths $(2 \%)$ related to rebleeding, which the authors suspected were attributable to undiagnosed aneurysms. In a study of 89 patients with angiogram-negative SAH, Whiting et al. ${ }^{29}$ reported that $25 \%$ had early hydrocephalus requiring ventriculostomy and that $13 \%$ required placement of a shunt. Symptomatic vasospasm was reported in 4 patients (4\%), 2 of whom developed associated infarctions. Three patients (3\%) died, and each of these patients was moribund on presentation.

No deaths and no rebleeding episodes occurred in the present study. Hydrocephalus requiring ventriculostomy was present in $28 \%$ of patients. Hydrocephalus was significantly more common in patients with the classic pattern of hemorrhage and in those with IVH (Tables 3 and 4). Four patients in this study, 3 with the classic pattern of hemorrhage and 1 with $\mathrm{PMH}$, could not be weaned from their ventriculostomy and required placement of a VP shunt.

Delayed cerebral ischemia due to vasospasm occurred in 4 patients $(7 \%)$ in this series and resulted in permanent ischemic changes in 1 patient (1.8\%). In the literature, the terms "symptomatic vasospasm" and "delayed cerebral ischemia" are often used interchangeably. Acknowledging that there may be some difference in the definitions for these terms, we found that the incidence of delayed cerebral ischemia and/or symptomatic vasospasm in patients with no identifiable cause of SAH ranges between $0 \%$ and $6 \% .3,4,9,25,29$ Several studies have shown that the most important risk factor for the development of delayed cerebral ischemia and/or symptomatic vasospasm is the amount of blood in the subarachnoid space. ${ }^{6,19,30,31}$

Hemorrhage volumes tend to be greater in the classic pattern of SAH, and, not surprisingly, delayed cerebral ischemia occurs primarily in patients with this type of $\mathrm{SAH}$. Vasospasm is rare in patients with $\mathrm{PMH}$, and when present, it tends to resolve without significant neurological deficits. Canhão et al. ${ }^{3}$ reported a $5.7 \%$ incidence of delayed ischemic deficits due to vasospasm in 35 patients with angiographically negative SAH with the classic hemorrhage pattern and no incidence in the PMH group. In a recent review of this topic, Gross and colleagues ${ }^{7}$ noted that for patients with the classic hemorrhage pattern the rate of delayed ischemic deficits was $9.7 \%$, while in patients with PMH the rate was $2.4 \%$. In the present study, delayed ischemic deficits occurred only in patients with the classic pattern of hemorrhage.

Clinical outcome following SAH with no identifiable source also appears to be related to the volume and type of hemorrhage, with worse outcomes reported for patients with the classic diffuse pattern of hemorrhage. ${ }^{8,11,20,22}$ In a series of 94 patients, Hui et al. ${ }^{10}$ noted that ultimately only $76 \%$ of patients with classic hemorrhage achieved 


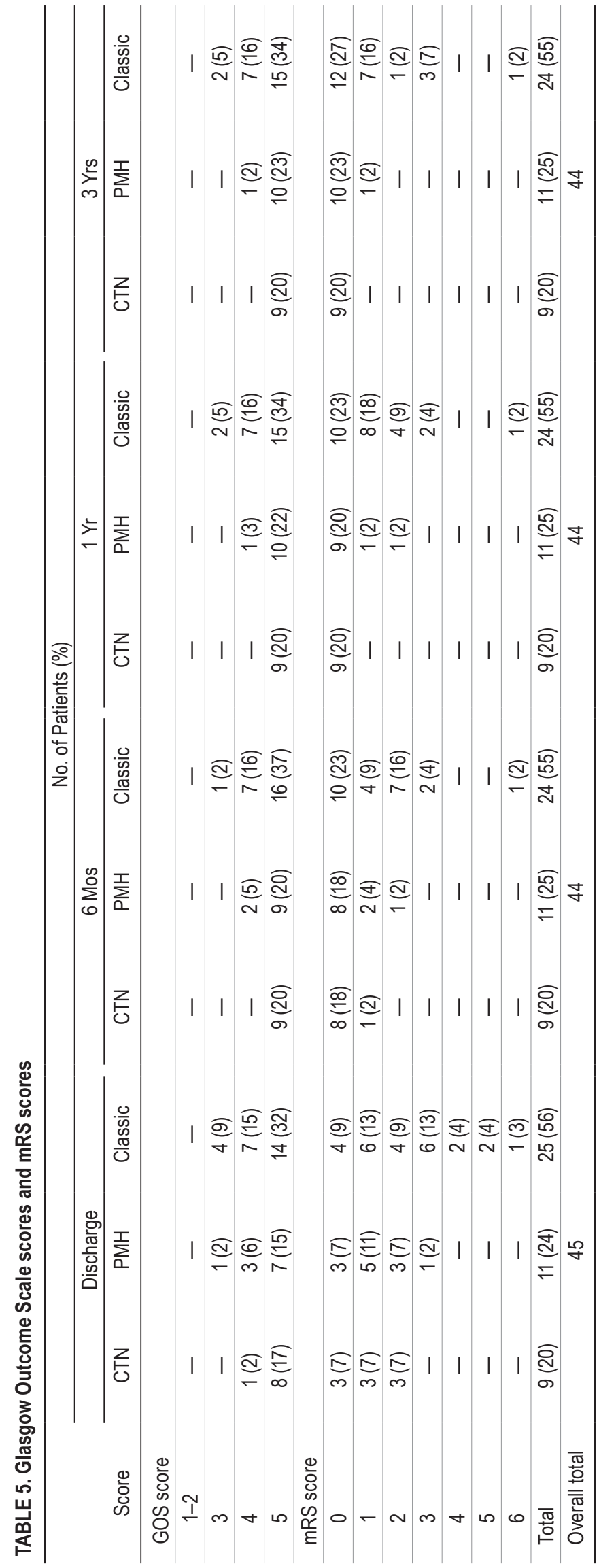




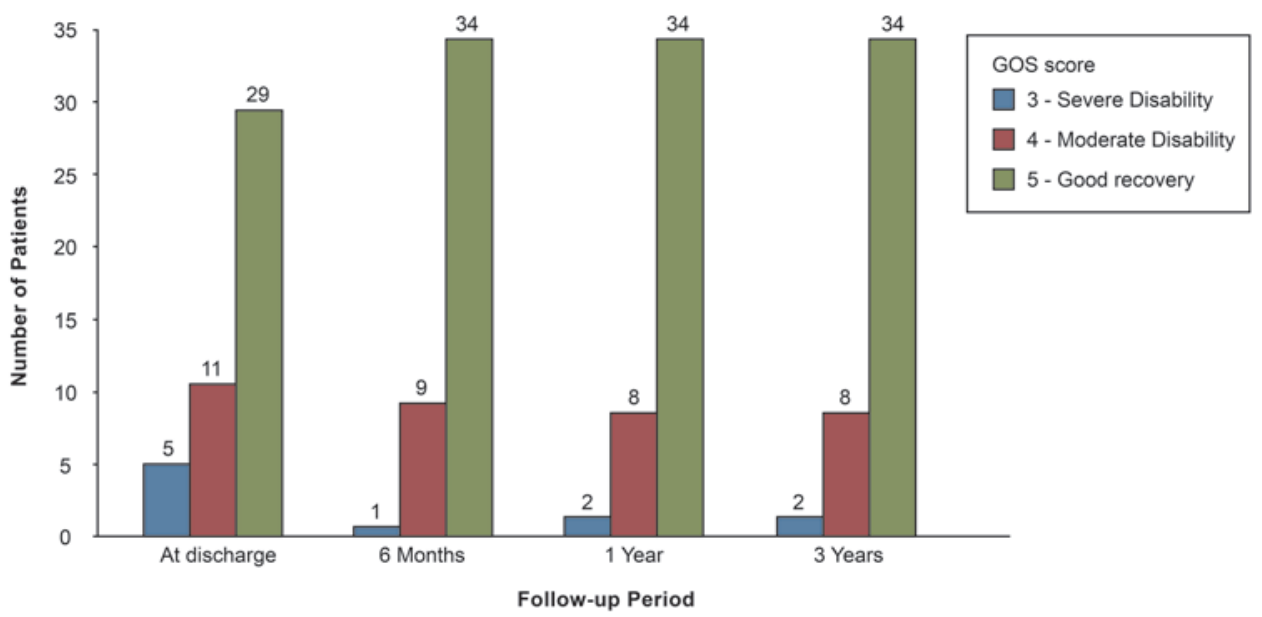

FIG. 2. Graph representing the GOS scores in patients without an identifiable source of SAH, from discharge to 3 years' follow-up. Figure is available in color online only.

complete independent recovery, as compared with $97 \%$ of patients with PMH. ${ }^{10}$ We found a similar pattern in our patients: at 6 months posthemorrhage (Table 5), only 58\% $(n=14)$ of the 24 patients with classic hemorrhage had recovered to an mRS score of 0 or 1, as compared with $95 \%$ $(n=19)$ of the 20 patients with CTN SAH or PMH. At 3 years posthemorrhage, the percentage of patients with an mRS score of 0 or 1 had increased to $79 \%(n=19)$ in the group of 24 with classic hemorrhage, although there were still 3 patients $(12.5 \%)$ with moderate disability requiring some help with activities of daily living.

A number of risk factors have been associated with an SAH whose source cannot be ascertained, including hypertension, chronic obstructive pulmonary disease, venous hypertension, diabetes, alcoholism, and drug abuse, but recurrent hemorrhage is rare, making a correlation difficult to establish. The absence of a clear explanation for hemorrhage in these patients raises concerns that they may har- bor occult vascular lesions or may have an increased risk of developing aneurysms or other vascular malformations. Angiographic follow-up in this group has been limited in previous studies. ${ }^{14,20,25,29}$

In Topcuoglu et al.'s ${ }^{25}$ series of 86 patients, follow-up angiography studies were obtained within the first 4 weeks after a patient's initial bleed, and only 4 patients were found to have aneurysms that had not been diagnosed on initial angiography. All 4 patients had the classic pattern of bleeding, and the lesion was diagnosed on the second angiography (3 patients) or the third (1 patient), which was performed within the first 4 weeks postbleed. Jung and colleagues ${ }^{14}$ reported similar findings in their series, identifying an aneurysm in only $1(1.5 \%)$ of 65 patients with PMH undergoing repeat 4-vessel angiography, versus 17 (46\%) of 37 patients with a classic pattern of hemorrhage. Little et al..$^{15}$ reported that repeat angiography performed between 1 and 6 weeks posthemorrhage demonstrated an

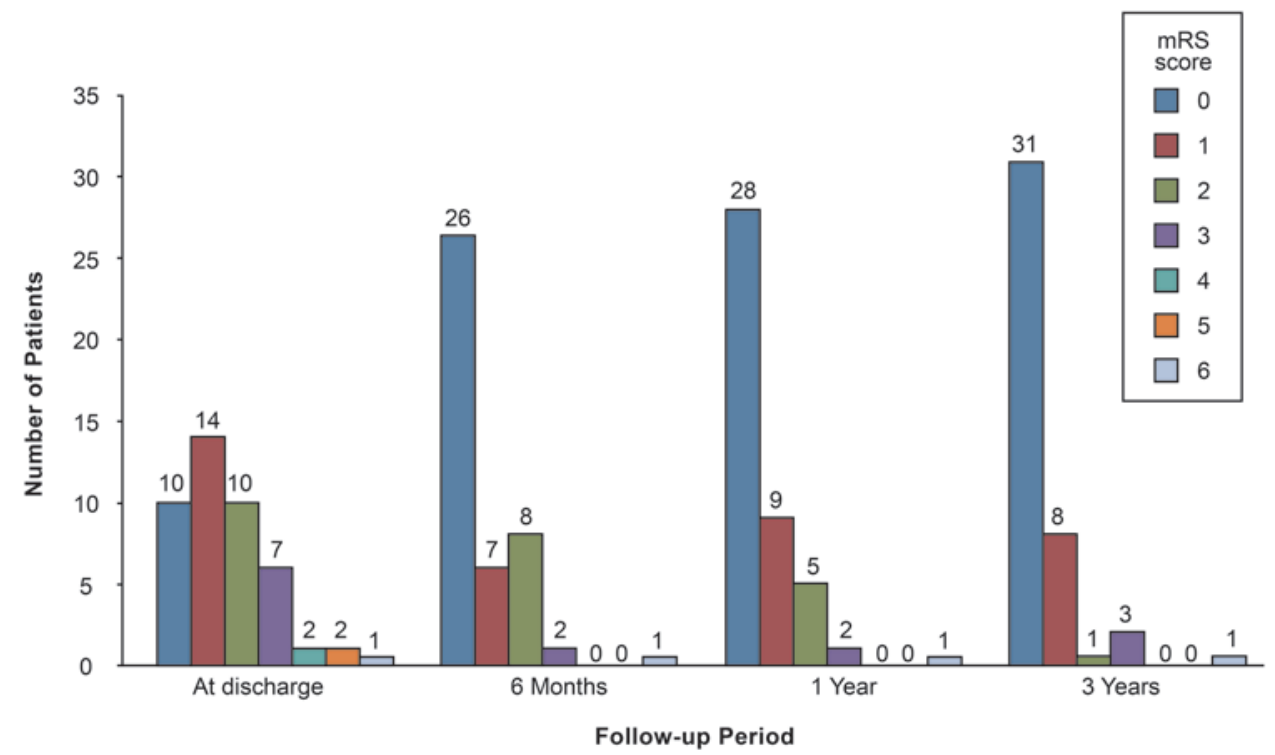

FIG. 3. Graph representing the mRS scores in patients without an identifiable source of SAH, from discharge to 3 years' follow-up. Figure is available in color online only. 
aneurysm in $5(12 \%)$ of 42 patients with a classic pattern of hemorrhage, in $1(7 \%)$ of 15 patients with $\mathrm{PMH}$, and in no patients with CTN.

In the present study, we prospectively followed a cohort of 56 patients with SAH whose diagnostic workup was negative at 4-6 weeks posthemorrhage. Long-term outcome was available at 6 months, 1 year, and 3 years in 44 of 56 patients. No cases of delayed hemorrhage were identified in any patient in this group; however, 1 patient died of unrelated causes at 4 months, and data were unavailable for 10 patients lost to follow-up. Angiographic follow-up at 3 years was negative for aneurysm, AVM, or other vascular abnormalities in 33 of the 44 available patients; the remaining 11 patients declined requests for repeat angiographic evaluation.

\section{Study Strengths and Limitations}

The main strengths of this study are 1) the availability of long-term clinical and angiographic follow-up for patients with SAH whose source was unknown, 2) and the fact that the entire follow-up was prospectively performed as part of the BRAT, which included clinical evaluations at 6 months, 1 year, and 3 years and angiographic followup at 3 years. However, the study has 3 limitations: 1) it was limited to a relatively small number of patients (56 patients); 2) data analysis was undertaken retrospectively; and 3) 10 patients (18\%) were lost to follow-up, and 11 of the 44 patients available for clinical follow-up declined to undergo additional imaging studies. While the size of the study population is limited, the results support conclusions reported by others that patients with SAH whose source is unknown, even those presenting with a classic diffuse pattern of hemorrhage, tend to have a good outcome. ${ }^{3,8,11,14}$

The potential for selection bias is an issue in any retrospective analysis but is limited in this cohort, as all patients were enrolled in the BRAT protocol at the time of their initial presentation and were prospectively monitored by a dedicated research nurse practitioner who acted as the study coordinator, monitored patient accrual, and was responsible for contacting patients for follow-up and for assessing $\mathrm{mRS}$ and GOS scores.

The loss of patients to follow-up can lead to significant bias in clinical outcome, particularly if these patients represent a subgroup with better or worse outcomes than those in the rest of the study population. Ten (18\%) of 56 patients were lost to follow-up in the present study; all 10 were discharged to home. Although 3 patients in this group required placement of an EVD, none required a shunt. At discharge, 8 patients had a GOS score of 5 , and 2 had a GOS score of 4 . Assuming that the GOS scores remained static, inclusion of these 10 patients would not have significantly altered clinical outcome at 3 years: GOS Score 3, $4 \%$ versus $5 \%$; GOS Score $4,19 \%$ versus $18 \%$; GOS Score $5,78 \%$ versus $77 \%$, respectively, for results with and without the additional 10 patients. Although delayed recurrent hemorrhage in patients with SAH of unknown etiology is considered rare, the possibility of such an event adversely affecting outcome in this group cannot be completely dismissed.

Finally, 11 of the 44 patients seen at the 3-year followup declined to undergo additional angiographic evaluation of any type. The lack of angiographic follow-up in these patients places some additional limits on the strength of our conclusions; however, none of these patients reported any signs or symptoms consistent with SAH.

\section{Conclusions}

The results of this study suggest that long-term angiographic follow-up beyond 6 weeks has little utility in patients with SAH of unknown etiology, regardless of the hemorrhage pattern, and that delayed rebleeding is an unexpected event. Although the clinical course of patients with SAH whose source is unknown is generally benign, vasospasm and hydrocephalus can occur, and close monitoring of patients who present with higher grade hemorrhages is indicated. Functional deficits occur primarily in patients with the classic pattern of hemorrhage and tend to improve over time.

\section{References}

1. Bederson JB, Connolly ES Jr, Batjer HH, Dacey RG, Dion JE, Diringer MN, et al: Guidelines for the management of aneurysmal subarachnoid hemorrhage: a statement for healthcare professionals from a special writing group of the Stroke Council, American Heart Association. Stroke 40:994-1025, 2009

2. Bonita R, Beaglehole R: Recovery of motor function after stroke. Stroke 19:1497-1500, 1988

3. Canhão P, Ferro JM, Pinto AN, Melo TP, Campos JG: Perimesencephalic and nonperimesencephalic subarachnoid haemorrhages with negative angiograms. Acta Neurochir (Wien) 132:14-19, 1995

4. Duong H, Melançon D, Tampieri D, Ethier R: The negative angiogram in subarachnoid haemorrhage. Neuroradiology 38:15-19, 1996

5. Farrés MT, Ferraz-Leite H, Schindler E, Mühlbauer M: Spontaneous subarachnoid hemorrhage with negative angiography: CT findings. J Comput Assist Tomogr 16:534-537, 1992

6. Fisher CM, Kistler JP, Davis JM: Relation of cerebral vasospasm to subarachnoid hemorrhage visualized by computerized tomographic scanning. Neurosurgery 6:1-9, 1980

7. Gross BA, Lin N, Frerichs KU, Du R: Vasospasm after spontaneous angiographically negative subarachnoid hemorrhage. Acta Neurochir (Wien) 154:1127-1133, 2012

8. Gupta SK, Gupta R, Khosla VK, Mohindra S, Chhabra R, Khandelwal N, et al: Nonaneurysmal nonperimesencephalic subarachnoid hemorrhage: is it a benign entity? Surg Neurol 71:566-572, 2009

9. Hayward RD: Subarachnoid haemorrhage of unknown aetiology. A clinical and radiological study of 51 cases. J Neurol Neurosurg Psychiatry 40:926-931, 1977

10. Hui FK, Tumialán LM, Tanaka T, Cawley CM, Zhang YJ: Clinical differences between angiographically negative, diffuse subarachnoid hemorrhage and perimesencephalic subarachnoid hemorrhage. Neurocrit Care 11:64-70, 2009

11. Ildan F, Tuna M, Erman T, Göçer AI, Cetinalp E: Prognosis and prognostic factors in nonaneurysmal perimesencephalic hemorrhage: a follow-up study in 29 patients. Surg Neurol 57:160-166, 2002

12. Jain VK, Hedge T, Easwaran RK, Das BS, Reddy GN: Benign subarachnoid haemorrhage (subarachnoid haemorrhage of unknown aetiology). Acta Neurochir (Wien) 86:89-92, 1987

13. Jennett B, Bond M: Assessment of outcome after severe brain damage. Lancet 1:480-484, 1975

14. Jung JY, Kim YB, Lee JW, Huh SK, Lee KC: Spontaneous 
subarachnoid haemorrhage with negative initial angiography: a review of 143 cases. J Clin Neurosci 13:1011-1017, 2006

15. Little AS, Garrett M, Germain R, Farhataziz N, Albuquerque FC, McDougall CG, et al: Evaluation of patients with spontaneous subarachnoid hemorrhage and negative angiography. Neurosurgery 61:1139-1151, 2007

16. Lloyd-Jones D, Adams R, Carnethon M, De Simone G, Ferguson TB, Flegal K, et al: Heart disease and stroke statistics-2009 update: a report from the American Heart Association Statistics Committee and Stroke Statistics Subcommittee. Circulation 119:e21-e181, 2009 (Errata in Circulation 119:e182, 2009; Circulation 122:e11, 2010; Circulation 124:e424, 2011)

17. McDougall CG, Spetzler RF, Zabramski JM, Partovi S, Hills NK, Nakaji P, et al: The Barrow Ruptured Aneurysm Trial. J Neurosurg 116:135-144, 2012

18. Pinto AN, Ferro JM, Canhão P, Campos J: How often is a perimesencephalic subarachnoid haemorrhage CT pattern caused by ruptured aneurysms? Acta Neurochir (Wien) 124:79-81, 1993

19. Rabb CH, Tang G, Chin LS, Giannotta SL: A statistical analysis of factors related to symptomatic cerebral vasospasm. Acta Neurochir (Wien) 127:27-31, 1994

20. Rinkel GJ, Wijdicks EF, Hasan D, Kienstra GE, Franke CL, Hageman LM, et al: Outcome in patients with subarachnoid haemorrhage and negative angiography according to pattern of haemorrhage on computed tomography. Lancet 338:964968, 1991

21. Rinkel GJ, Wijdicks EF, Vermeulen M, Ramos LM, Tanghe HL, Hasan D, et al: Nonaneurysmal perimesencephalic subarachnoid hemorrhage: CT and MR patterns that differ from aneurysmal rupture. AJNR Am J Neuroradiol 12:829-834, 1991

22. Ruigrok YM, Rinkel GJ, Van Gijn J: CT patterns and longterm outcome in patients with an aneurysmal type of subarachnoid hemorrhage and repeatedly negative angiograms. Cerebrovasc Dis 14:221-227, 2002

23. Schwartz TH, Mayer SA: Quadrigeminal variant of perimesencephalic nonaneurysmal subarachnoid hemorrhage. Neurosurgery 46:584-588, 2000

24. Spetzler RF, McDougall CG, Albuquerque FC, Zabramski JM, Hills NK, Partovi S, et al: The Barrow Ruptured Aneurysm Trial: 3-year results. J Neurosurg 119:146-157, 2013 (Erratum in J Neurosurg 120:581, 2014)

25. Topcuoglu MA, Ogilvy CS, Carter BS, Buonanno FS, Koroshetz WJ, Singhal AB: Subarachnoid hemorrhage without evident cause on initial angiography studies: diagnostic yield of subsequent angiography and other neuroimaging tests. J Neurosurg 98:1235-1240, 2003

26. van Gijn J, Rinkel GJ: Subarachnoid haemorrhage: diagnosis, causes and management. Brain 124:249-278, 2001

27. Vergouwen MD, Vermeulen M, van Gijn J, Rinkel GJ, Wijdicks EF, Muizelaar JP, et al: Definition of delayed cerebral ischemia after aneurysmal subarachnoid hemorrhage as an outcome event in clinical trials and observational studies: proposal of a multidisciplinary research group. Stroke 41:2391-2395, 2010

28. Vermeer SE, Rinkel GJ, Algra A: Circadian fluctuations in onset of subarachnoid hemorrhage. New data on aneurysmal and perimesencephalic hemorrhage and a systematic review. Stroke 28:805-808, 1997

29. Whiting J, Reavey-Cantwell J, Velat G, Fautheree G, Firment $\mathrm{C}$, Lewis S, et al: Clinical course of nontraumatic, nonaneurysmal subarachnoid hemorrhage: a single-institution experience. Neurosurg Focus 26(5):E21, 2009

30. Wilson DA, Nakaji P, Abla AA, Uschold TD, Fusco DJ, Oppenlander ME, et al: A simple and quantitative method to predict symptomatic vasospasm after subarachnoid hemorrhage based on computed tomography: beyond the Fisher scale. Neurosurgery 71:869-875, 2012

31. Zabramski JM, Spetzler RF, Bonstelle C: Chronic cerebral vasospasm: effect of volume and timing of hemorrhage in a canine model. Neurosurgery 18:1-6, 1986

\section{Author Contributions}

Conception and design: Zabramski, Spetzler. Acquisition of data: Elhadi, Mendes. Analysis and interpretation of data: Elhadi, Almefty. Drafting the article: Zabramski, Elhadi, Almefty. Critically revising the article: Zabramski, Elhadi, Nakaji. Reviewed submitted version of manuscript: Zabramski, McDougall, Albuquerque. Statistical analysis: Elhadi, Almefty. Administrative/technical/material support: Zabramski, Albuquerque, Preul. Study supervision: Zabramski, McDougall, Preul, Spetzler.

\section{Correspondence}

Joseph M. Zabramski, c/o Neuroscience Publications, Barrow Neurological Institute, St. Joseph's Hospital and Medical Center, 350 W. Thomas Rd., Phoenix, AZ 85013. email: neuropub@ dignityhealth.org. 